\title{
Shock Graph and Shape matching
}

\author{
${ }^{1}$ Dr.B.Tamil Selvi, ${ }^{2}$ S.Deepika \\ ${ }^{1}$ Department of Mathematics, P.S.G.R Krishnammal College for Women \\ Coimbatore. Tamilnadu, India \\ ${ }^{2}$ Department of Mathematics, P.S.G.R Krishnammal College for Women \\ Coimbatore. Tamilnadu, India
}

\begin{abstract}
The aim of this paper to develop a theory for generic representation of 2-D shape, where structural descriptions are derived from the shocks (singularities) of a curve evolution process, acting on bounding contours. The shocks are organized into a directed, acyclic shock graph and complexity is managed by attending to the most significant (central) shape components first. The space of all such graphs is highly structured and can be characterized by the rules of a shock graph grammar which permits a reduction of a shock graph to a unique rooted shock tree. A novel tree matching algorithm which finds the best set of corresponding nodes between two shock trees in polynomial time.
\end{abstract}

Keywords: shape matching, shock graph, shock graph grammar, subgraph isomorphism

\section{Introduction:}

The system of shocks derived from the curve evolution process into a graph, which is defined as the shock graph or SG. The shock types will label each vertex in the graph and the shock formation times will direct edges to provide an ordering for matching and a basis for subgraph approximation.

Blum's skeleton is area-based and provides a description of shapes via the loci of centers of covering balls. Variations on this theme include smoothed local symmetries (Brady and Asada, 1984) and the process inferring symmetric axis (Leyton,1988). The skeleton has the advantage of providing a different (from Fu) type of graph on which to base matching, but again sensitivity causes problems. Proper skeletons can be found interactively, but not automatically.

In this paper shock graphs of order one, two, three and four are discussed. The graphs are characterized by shock graph grammar which reduces shock graph to unique rooted shock tree and algorithm is discussed for finding best set of corresponding nodes between two shock trees in polynomial time.

Particular shapes can vary in detail from one another, variations between shapes derive from an organization of these particular shapes into equivalence classes. Specifically, for simple closed curves in the plane the following evolution equation was studied.

$C_{t}=(1+\alpha k) N$

$$
\mathbf{C}(\mathbf{s}, \mathbf{0})=\mathbf{C}_{0}(\mathrm{~s})
$$

Here $\mathbf{C}(\mathbf{s}, \mathbf{t})$ is the vector of curve coordinates, $\mathbf{N}(\mathbf{s}, \mathbf{t})$ is the inward normal, is the path parameter and $\mathbf{t}$ is the evolutionary time of the deformation. The constant $\boldsymbol{\alpha} \geq \mathbf{0}$ controls the regularizing effects of curvature $\mathrm{k}$. When $\alpha$ is large, the equation becomes a geometric heat equation, when $\boldsymbol{\alpha}=\mathbf{0}$, the equation is equivalent to Blum's grassfire transformation.

\section{Preliminary Notes}

Definition 2.1: At a 1-shock the radius function varies monotonically, as is the case for a protrusion. At a 2shock the radius function achieves a strict local minimum such that the medial axis is disconnected when the shock is removed, e.g., at a neck. At a 3-shock the radius function is constant along an interval, e.g., for a bend with parallel sides. At a 4-shock the radius function achieves a strict local maximum, as is the case when the evolving curve annihilates into a single point or a seed. TheLoci of these shocks give blum'smedialaxis.
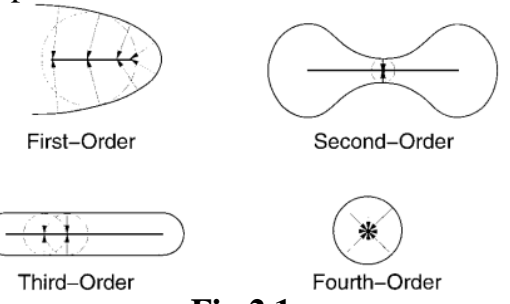

Fig 2.1

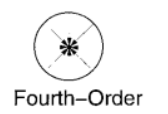


Definition 2.2: Consider the above figure, the coloring can be formalized as follows. Let $\mathrm{X}$ be the open interior of a simple closed curve and $\mathrm{Me}(\mathrm{X})$ its medial axis (the set of points reached simultaneously by two or more fire fronts). Let $\mathrm{B}(\mathrm{x}, \epsilon)$ be an open disc of radius $\epsilon$ centered at $\mathrm{x} \in$ Xand let $\mathrm{R}(\mathrm{x})$ denote the radius of the largest such disk contained in $\mathrm{X}$. Let $\mathrm{N}(\mathrm{x}, \epsilon)=\mathrm{Me}(\mathrm{X}) \cap \mathrm{B}(\mathrm{x}, \epsilon) \backslash\{\mathrm{x}\}$ define punctured $\epsilon-$ neighbourhood of $x$, one that does not contain $x$ itself. A medial axis point $x \in \operatorname{Me}(x)$ is

1. type 4 if $\exists \epsilon>0$ st. $R(x)>R(y) \forall y \in N(x, \epsilon)$.

2. type 3 if $\exists \epsilon>0$ st. $R(x)=R(y) \forall y \in N(x, \epsilon)$ and $N(x, \epsilon) \neq \emptyset$.

3. type 2 if $\exists \epsilon>0$ st. $R(x)=R(y) \forall y \in N(x, \epsilon)$ and $N(x, \epsilon) \neq \emptyset$ and $N(x, \epsilon)$ is not connected.

4. type 1 otherwise.

Definition 2.3: A 2-D shape Shock Graph is the bounded interior of a simple closed (Jordan) curve.

From the coloring of shocks into four types, it can be seen that 2-shocks and 4-shocks are isolated points, whereas 1-shocks and 3-shocks are neighbored by other shocks of the same type. So, we denote the groups with labels $\tilde{1}, 2, \widetilde{3}$, 4and breaking apart the $\tilde{1}$ 's at branch-points. Let \# denote a start symbol and $\phi$ a terminal symbol. The SG is a connected graph, rooted at a vertex labeled \#, such that all other (non-terminal) vertices are shock groups and directed edges to non-terminal vertices indicate the genesis of new shock groups.

Definition 2.4: The Shock Graph of a 2-D shape, SG is a labeled graph $G=(V, E, \gamma)$ with vertices $\mathbf{V}=\{1 \ldots n\}$, edges $(i, j) \in E \subseteq V \times V$ directed from vertex $i$ to vertex $j$ if and only if $i \neq j, t_{i} \geq t_{j}$ and $i \cup j$ is connected in the plane. labels $\gamma: V \rightarrow 1$ with $1 \in\{\tilde{1}, 2, \tilde{3}, 4, \#, \phi\}$, topology such that $\forall j \in V$ with $\gamma(j) \neq \#, \exists i \in V$ with $(i, j) \in E$.

Definition 2.5: Shock Graph Grammar or SGG is a quadruple $G=\left(V, \sum, R, S\right)$, with

1. $\mathrm{V}=\{\tilde{1}, 2, \tilde{3}, 4, \#, \phi\}$, the alphabet,

2. $\Sigma=\{\phi\}$, the set of terminals,

3. $\mathrm{S}=$ \# the start symbol,

4. $\quad R=\left\{R_{1}, \ldots \ldots \ldots R_{10}\right\}$, the set of rules in the Fig 2.1.

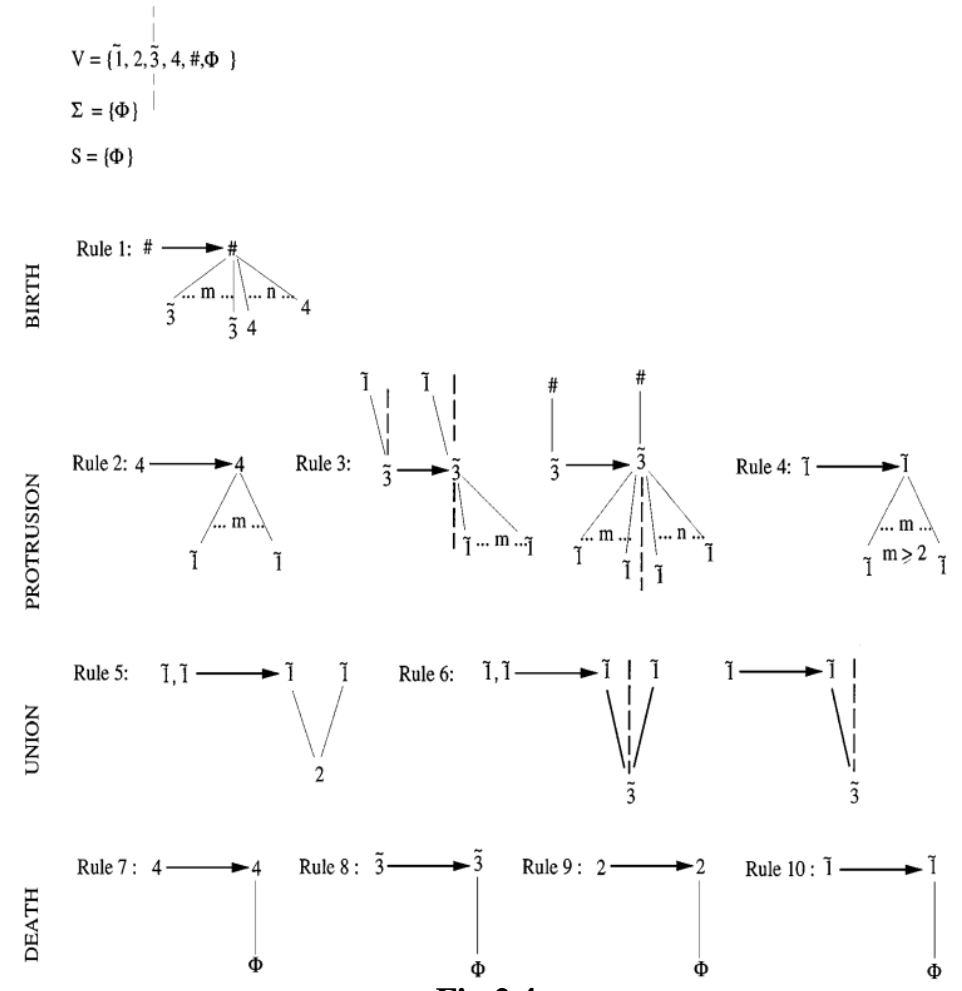

Fig 2.4

The Shock Graph Grammar, SGG. Dashed lines partition distinct ends of a $\widetilde{3}$. The rules are grouped according to the differentsemantic processes (on the left) that they characterize. Note that the grammar is not context-free e.g., rule 3 indicates that a $\tilde{1}$ can only be addedonto an end of a $\widetilde{3}$ that has no parent $\tilde{1}$.

Definition 2.6: Given two graphs $G=\left(V_{1}, E_{1}\right)$ and $H=\left(V_{2}, E_{2}\right)$, find the maximum integer $k$, such that there exists two subsets of cardinality $\mathrm{k}, \mathrm{E}_{1}^{\prime} \subseteq \mathrm{E}_{1}$ and $\mathrm{E}_{2}^{\prime} \subseteq \mathrm{E}_{2}$ and the induced subgraphs (not necessarily connected) $\mathrm{G}^{\prime}=\left(\mathrm{V}_{1}, \mathrm{E}_{1}^{\prime}\right)$ and $\mathrm{H}^{\prime}=\left(\mathrm{V}_{2}, \mathrm{E}_{2}^{\prime}\right)$ are isomorphic. 
Definition 2.7: Let $\Psi(\widehat{S})$ and $\Psi\left(\mathrm{S}^{\prime}\right)$ denote the interpolated 4-D curves passing through the points of the sets $\widehat{S}$ and $S^{\prime}$ respectively. A Hausdorff distance measure between the curves $\Psi(\widehat{S})$ and $\Psi\left(S^{\prime}\right)$ is defined by finding the closest point on curve $\Psi$ (S') for each point in the sequence $\widehat{S}^{\prime}$ and the closest point on curve $\Psi(\widehat{S})$ for each point in the sequence $S^{\prime}$.

$\Delta\left(\Psi(\widehat{S}), \Psi\left(S^{\prime}\right)\right)=\sum_{\varsigma \in \hat{S}} \inf _{\eta \in \Psi\left(S^{\prime}\right)}\left\|_{\varsigma}-\eta\right\|_{2}+\sum_{\varsigma \in S} \underset{\eta \in \Psi(\hat{S})}{\inf }\left\|_{\varsigma}-\eta\right\|_{2}$.

Definition 2.8: Let $\mathrm{G}=\left(\mathrm{V}_{1}, \mathrm{E}_{1}\right)$ and $\mathrm{H}=\left(\mathrm{V}_{2}, \mathrm{E}_{2}\right)$ be two shock graphs to be matched, with $\mathrm{n}_{1}$ and $\mathrm{V}_{2}=\mathrm{n}_{2}$. Define $\mathrm{d}$ to be the maximum degree of any vertex in $\mathrm{G}$ and $\mathrm{H}$,

$\delta(\mathrm{G}), \delta(\mathrm{H}))$.For each vertex $\mathrm{v}$, we define $\chi(\mathrm{v}) \in \mathrm{R}^{\mathrm{d}-1}$ as the unique eigen decomposition vector.

III. Shock Graph and Shape Matching

\section{Theorems, Algorithm and Example}

\section{Theorem 3.1}

Any 2-D shape graph has a unique corresponding shock graph SG.

\section{Proof:}

The uniqueness of the skeleton $\mathrm{S}(\mathrm{X})$ follows from its definition as the union of maximum open discs. Hence the medial axis $\mathrm{Me}(\mathrm{X})$, which is strictly contained in the skeleton $\mathrm{S}(\mathrm{X})$ is also unique. The coloring of medial axis points into four types is unique, which implies that a unique set of vertices exists for the corresponding SG.

Finally, by Definition 2.4, the direction of an edge between two abutting vertices is ambiguous only when $t_{i}=t_{j}$ for all shocks in $I$ and $j$. Due to the continuity of the radius function along the skeleton, the only possibility is that the two vertices share the point where they touch, in that case we have the contradiction that all shocks in $i$ and $j$ would lie in the same $\overline{3}$ and hence in a single vertex. The uniqueness of the shock graph follows.

Hence the theorem.

\subsection{The shock graph Grammar}

The notion of entry-level categories for shape that is intimately connected to the topological structure of the shock graph. This structure is highly constrained because the events that govern the birth, combination and death of shock groups can be abstracted into a small number of rewrite rules as shown in Fig. 2.1.

\section{Lemma 3.3}

A vertex in the SG can have at most two $\widetilde{1}$ 's as parents. If it has exactly two $\widetilde{1}$ 's as parents, it must be a 2-shock or a $\widetilde{3}$.

Proof:

Recall that a $\widetilde{1}$ is a curve segment of 1 -shocks along which the radius function varies monotonically.

Let a $\widetilde{1}$ of length $L$ be parameterized by arc-length $s$, with $s \in(0,1]$, such that the radius function $R(s)$ increases monotonically with $\mathrm{s}$. For each $\mathrm{s} \in(0,1]$, there exists a continuous mapping from $\mathrm{x}(\mathrm{s})$ to its associated pair of bitangent points $(\mathrm{p}(\mathrm{s}), \mathrm{q}(\mathrm{s}))$, by which the two boundary segments associated with the interval $(0, \mathrm{~L}]$ can be reconstructed, see Fig. 3.1(a).

Let $\alpha(\mathrm{s})$ be the angle between the line segments $\mathrm{p}(\mathrm{s}) \mathrm{x}(\mathrm{s})$ and $\mathrm{q}(\mathrm{s}) \mathrm{x}(\mathrm{s})$, on the narrower side. Two conditions must hold,

1) $\alpha(s)$ must be $\leq \pi$ for each $s \in(0,1]$, since the radius function $R(s)$ increases monotonically with s.

2) No other shock can lie in the reconstructed (shaded) region in Fig. 3.1(a), because the grassfire can only traverse a point in the plane once.

Now, because the radius function along the $\tilde{1}$ increases with sand the radius function is continuous along the skeleton, $x(0)$ cannot itself be, or abut, a 4-shock. Let us assume that $\mathrm{x}(0)$ abuts the first point (where the radius function is smallest) of a second (distinct) $\tilde{1}$.

The above two conditions must hold for the second $\widetilde{1}$. Therefore, the only possibility is that $\alpha\left(0^{+}\right)$is identically $\pi$ for points infinitesimally close to $\mathrm{x}(0)$ on each $\tilde{1}$.Hence, there exists an $\epsilon>0$ such that the open disc of radius ecentered at $x(0)$ is strictly contained in the union of the shaded regions reconstructed by $x(0)$ and the two $\tilde{1}$ 's (see Fig. 3.1(a)). This implies that the first point of no other $\widetilde{1}$ can abut $x(0)$. We note that by coloring a 2 shock, as illustrated in Fig 3.1(b) (top). A second possibility is that $x(0)$ abuts the endpoint of a $\tilde{3}$. By a similar argument as the one above, there can be at most one such $\tilde{3}$. In the event that the other end of the $\tilde{3}$. Abutsthe first point (where the radius function is smallest) of a distinct $\tilde{1}$, the $\tilde{3}$ is the child of these two $\tilde{1}$ 's. 


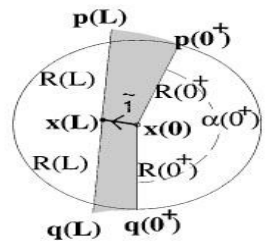

(a)

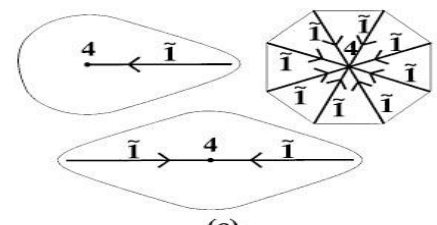

(c)

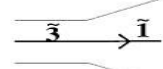

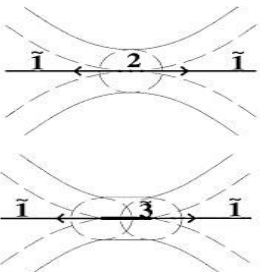

(b)

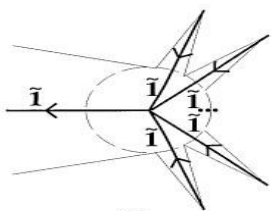

(d)

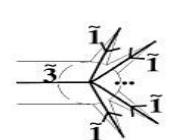

(e)

Fig 3.1

An illustration of shock patterns, with arrows drawn in the direction of increasing radius.

(a) Geometry of a $\tilde{1}$.

(b) Two $\tilde{1}$ 's flow outwards from a 2 or $\tilde{3}$.

(c) An integer number of $\tilde{1}$ 's may flow into 4 .

(d) Two or more $\tilde{1}$ 's may flow into $\tilde{1}$.

(e) An end-point of a 3̃may have a single $\tilde{1}$ flowing out of it, no shocks flowing into or out of it or an integer number of $\tilde{1}$ 's flowing into it.

\section{Theorem 3.4}

The rewrite rules of the SGG are sufficient to derive the shock graph SG of any 2-D shape shock graph.

Proof:

The strategy is to derive the rules by enumerating all legal parents and children for each vertex type. To determine the remaining local configurations it is now sufficient to enumerate all the possible children for each vertex type. A child corresponds to an abutting vertex, containing no shocks that formed after any of the shocks in the vertex under consideration (2.4 Definition). We know that no 2, $\tilde{3}$ or 4 can have a 2, $\tilde{3}$ or 4 as a parent or as a child. If the vertex is of type $2, \tilde{3}$ or 4 , only children of type $\tilde{1}$ have to be considered.

Children of a \# :

Since the SG is built in "reverse" time, any children of the \# symbol will be the last shocks to form in the forward evolution. By coloring, an isolated point of annihilation is a 4, an annihilating curve segment is a $\tilde{3}$. These situations correspond to Rule 1 in Fig. 2.1, which states that any number of $\tilde{3}$ 's or 4's can act as seeds for the shape. These are the only possible children. A $\phi$ cannot be a child since the interior of a Jordan curve is nonempty and hence a null shape is disallowed.

\section{Children of a $\widetilde{\mathbf{1}}$ :}

A child of a $\tilde{1}$ can have no shock whose time of formation is greater than that of any shock in the $\tilde{1}$ (2.4 Definition). Hence, by coloring a child cannot be a 4. It is possible for two or more distinct $\tilde{1}$ 's to be children, e.g., one could place two or more triangular wedges around the dashed circle in Fig. 3.1(d). This corresponds to Rule 4. By Lemma 3.3, a 2 must be a child of two distinct $\tilde{1}$ 's. This corresponds to Rule 5. Referring to the proof of Lemma 3.3, a $\tilde{1}$ cannot have two or more $\tilde{3}$ 's as children.

However, a single $\tilde{3}$ could be a child, as in Fig. 3.1(e) (left). The other end of the $\tilde{3}$ may or may not be the child of a distinct $\widetilde{1}$. Thus, in contrast to Rule 5, we have two separate cases in Rule 6 . Finally, when a $\tilde{1}$ has no abutting shock with smaller radius, it has a8 as a child. This corresponds to Rule 10 .

\section{Children of a 2:}

If a 2 has a $\tilde{1}$ as a child, its time of formation must be greater than that of all shocks in the $\tilde{1}$ (2.4 Definition). However, this would violate the coloring in Fig. 3.1(b). Therefore, the only possible child is8, corresponding to Rule 9.

\section{Children of a $\widetilde{3}$ :}

In contrast to a $\tilde{1}$, which can only have children at the end point where the radius function is smallest (2.4 Definition), a $\tilde{3}$ can have children at either end point. It is possible for several $\tilde{1}$ 's to be children, e.g., one could 
place one or more triangular wedges around the dashed circle in Fig. 3.1(e) (right). However, by the same argument as in the proof of Lemma 3.3, children can only be present at an end point that does not have a $\tilde{1}$ as a parent. Hence, we have two cases in Rule 3, if one end point of a $\tilde{3}$ has a parent $\tilde{1}$, an integer number of $\tilde{1}$ 's can be children at the other end point, if neither end point has a parent $\tilde{1}$, an integer number of $\tilde{1}$ 's can be children at each end point. Finally, when the $\tilde{3}$ has no abutting shock with smaller radius at either end point, as in Fig. 3.1(e), it has a8 as a child. This corresponds to Rule 8 .

\section{Children of a 4 :}

By the coloring, 4 correspond to a medial axis point where the radius function achieves a strict local maximum. It may have an integer number of $\tilde{1}$ 's as children, examples of which appear in Fig. 3.1(c). This corresponds to Rule 2. When it has no abutting $\widetilde{1}$ 's, as in the case of a perfect circle, it has 8 as a child. This corresponds to Rule 7.The above enumeration of all legal local vertex configurations in the SG shows that its structure is highly constrained. In particular, since a rewrite rule exists in Fig. 2.1, for each legal parent/child of each vertex type.

Hence the theorem.

\subsection{Shock graph to Shock trees}

In this section we present a reduction that takes a DAG representing a shock graph to a unique vertex labeled rooted tree, whose size is polynomially bounded by the size of the original shock graph. To begin, let $G=(V, E)$ be a DAG representing a shock graph on $n$ vertices. A loop $\mathrm{L}$ is a subgraph of $\mathrm{G}$ formed by the intersection of two directed paths. More formally, $\mathrm{L}$ originates at a vertex $\mathrm{b}$, follows two paths $\mathrm{P}_{1}$ and $\mathrm{P}_{2}$ and ends at the vertex t. We denote $\mathrm{b}$ as the base of $\mathrm{L}$, the tip of LandP $\mathrm{P}_{1}$ and $\mathrm{P}_{2}$ the wings of $\mathrm{L}$. Referring to the protrusion and birth rewrite rules (rules 1, 2, 3 and 4) in Fig 2.1, the base of L can be a vertex whose type is drawn from the set $\{\#, 4, \tilde{3}, \tilde{1}\}$. The wings $\mathrm{P}_{1}$ and $\mathrm{P}_{2}$, are directed paths consisting of a sequence of vertices whose types are drawn from the set $\{4, \tilde{3}, \tilde{1}\}$ (rules $1,2,3,4$ and 6 ). Finally, the tip of $L$ can be a vertex of type either 2 or $\tilde{3}$ (rules 5 and 6).

Assume that the tip $\mathrm{t}$ of $\mathrm{L}$ is a vertex of type 2 . Then by rule $9, \mathrm{~L}$ will be terminated at a vertex labeled 8 . Next, if $t$ is a vertex of type $\tilde{3}$, then $P_{1}$ and $P_{2}$ represent two directed sequences of shocks that enter at opposite ends of t. In this case, $t$ cannot satisfy rule 3 and must be the root of a single node subgraph having label 8 (rule 8), therefore conclude that the tips of all loops are adjacent to nodes having type 8in G and that each such tip participates in exactly one loop.

In our reduction, for each such tip node $t$ we will maintain duplicate copies $t_{1}$ and $t_{2}$ and redefine $L$ to be the union of $\mathrm{b}$ and two new disjoint paths. $\mathrm{P}_{1}^{\prime}=\mathrm{P}_{1} \cup\left\{\mathrm{t}_{1}\right\} \cup\{\phi\}$ and $\mathrm{P}_{2}^{\prime}=\mathrm{P}_{2} \cup\left\{\mathrm{t}_{2}\right\} \cup\{\phi\}$. It is easy to see, by induction on the number of tips in $\mathrm{G}$, that such a reduction is

unique and produces a directed or equivalently a rooted tree. Further, since $\mathrm{G}$ has only $\mathrm{O}(\mathrm{n})$ tips, each of which is duplicated atmost once, there is an $\mathrm{O}(\mathrm{n})$ increase in size of the graph. To perform the reduction, we need only check the in-degree of any 3 's and 2's and duplicate them if necessary. The complete reduction is therefore a linear time process in terms of the number of vertices in G. The matcher will maintain two copies of every $\tilde{3}$ with at least one $\tilde{1}$ child on each side (rule 3), each with its respective children. As correspondences are found, a match of one such $\tilde{3}$ will force a match of its copy.

\subsection{Theorem}

For the sum of the first $\mathrm{k}$ eigen values of a symmetric matrix A, the following semidefinite programming characterization holds.

$\lambda_{1}(\mathrm{~A})+\cdots+\lambda_{\mathrm{k}}(\mathrm{A})=\max \mathrm{A}$. $\mathrm{U}$ such that $\operatorname{trace}(\mathrm{U})=\mathrm{k}$

or, in a dual setting

$$
0 \leq \mathrm{U} \leq \mathrm{I}
$$

$\lambda_{1}(\mathrm{~A})+\cdots+\lambda_{\mathrm{k}}(\mathrm{A})=\min \mathrm{kz}+\operatorname{trace}(\mathrm{V})$ such that $\mathrm{zI}+\mathrm{V} \geq \mathrm{A}, \mathrm{V} \geq 0$

Proof:

Before applying the above theorem, let us first convert our shock trees to adjacency matrices. Given a bounded degree, rooted tree $\mathrm{G}=(\mathrm{V}, \mathrm{E})$ with $|\mathrm{V}|=\mathrm{n}$ and $|\mathrm{E}|=\mathrm{m}$, define the adjacency matrix $\mathrm{A}$ of $\mathrm{G}$ to be a $\mathrm{n} \times \mathrm{n}$ symmetric, $\{0,1\}$ matrix with its $(i, j)^{\text {th }}$ entry $A_{i, j}$ equal to 1 if $(i, j) \in E$ and 0 otherwise. For each vertex $v \in G$, let $\delta(\mathrm{v})$ be the degree of $\mathrm{v}$ and let $\delta(\mathrm{G})$ be the maximum degree over all vertices in $\mathrm{G}$.

For every vertex $\mathrm{u} \in \mathrm{G}$, we define $\chi(u)$ to be a vector in $\mathrm{R}^{\delta(\mathrm{G})-1}$, obtained through the following procedure, For any child $\mathrm{v}$ of $\mathrm{u}$ in $\mathrm{G}$, construct the adjacency matrix $\mathrm{A}_{\mathrm{v}}$ of the induced subtree rooted at $\mathrm{v}$ and for $\mathrm{A}_{\mathrm{v}}$, compute the quantity $\lambda_{v}=\lambda_{1}\left(\mathrm{~A}_{\mathrm{v}}\right)+\cdots+\lambda_{\mathrm{k}}\left(\mathrm{A}_{\delta(\mathrm{v})-1}\right)\left(\mathrm{A}_{\mathrm{v}}\right)$. Construct $\chi(u)$ as the vector formed by $\left\{\lambda_{v_{1}} \ldots . \lambda_{v_{\delta(\mathrm{u})}}\right\}$ for which $\lambda_{\mathrm{v}_{1}} \geq \cdots \geq \lambda_{\mathrm{v}_{\delta(\mathrm{u})}}$.

\subsection{Algorithm for matching two Shock trees}

The algorithm recursively finds matches between vertices, starting at the root of the shock tree and proceeds down through the subtrees in a depth-first fashion. The notion of a match between vertices incorporates two key 
terms, first is a measure of the topological similarity of the subtrees rooted at the vertices, while the second is a measure of the similarity between the shock geometry encoded at each node.

Procedure isomorphism $(\mathrm{G}, \mathrm{H})$

$\phi(\mathrm{G}, \mathrm{H}) \leftarrow \emptyset$

$\mathrm{d} \leftarrow \max (\delta(\mathrm{G}), \delta(\mathrm{H}))$

for $\mathrm{u} \in \mathrm{V}_{\mathrm{G}}$ compute $\chi(u) \in \mathrm{R}^{\mathrm{d}-1}$

for $\mathrm{v} \in \mathrm{V}_{\mathrm{H}}$ compute $\chi(v) \in \mathrm{R}^{\mathrm{d}-1}$

call match $(\operatorname{root}(\mathrm{G}), \operatorname{root}(\mathrm{H}))$

$\operatorname{return}(\operatorname{cost}(\phi(\mathrm{G}, \mathrm{H}))$

end

procedure match $(\mathrm{u}, \mathrm{v})$

do

\{

Let $\mathrm{G}_{\mathrm{u}} \leftarrow$ rooted subtree of $\mathrm{G}$ at $\mathrm{u}$

Let $\mathrm{H}_{\mathrm{v}} \leftarrow$ rooted subtree of $\mathrm{H}$ at $\mathrm{v}$

compute $\left|\mathrm{V}_{\mathrm{G}_{\mathrm{u}}}\right| \times\left|\mathrm{V}_{\mathrm{H}_{\mathrm{v}}}\right|$

weight matrix $\prod\left(\mathrm{G}_{\mathrm{u}}, \mathrm{H}_{\mathrm{v}}\right)$

$\mathrm{M} \leftarrow$ max cardinality, minimum weight

bipartite matching in $\mathrm{G}\left(\mathrm{V}_{\mathrm{G}_{\mathrm{H}}}, \mathrm{V}_{\mathrm{H}_{\mathrm{V}}}\right)$

with weights from $\prod\left(\mathrm{G}_{\mathrm{u}}, \mathrm{H}_{\mathrm{v}}\right)$

$\left(\mathrm{u}^{\prime}, \mathrm{v}^{\prime}\right) \leftarrow$ minimum weight pair in $\mathrm{M}$

$\phi(\mathrm{G}, \mathrm{H}) \leftarrow \phi(\mathrm{G}, \mathrm{H}) \cup\left\{\left(\mathrm{u}^{\prime}, \mathrm{v}^{\prime}\right)\right\}$

call match $\left(\mathrm{u}^{\prime}, \mathrm{v}^{\prime}\right)$

$$
\begin{aligned}
& \mathrm{G}_{\mathrm{u}} \leftarrow \mathrm{G}_{\mathrm{u}}-\left\{\mathrm{x} \mid \mathrm{x} \in \mathrm{V}_{\mathrm{G}_{\mathrm{H}}} \text { and }(\mathrm{x}, \mathrm{w}) \in \phi(\mathrm{G}, \mathrm{H})\right\} \\
& \mathrm{H}_{\mathrm{v}} \leftarrow \mathrm{H}_{\mathrm{v}}-\left\{\mathrm{y} \mid \mathrm{y} \in \mathrm{V}_{\mathrm{H}_{\mathrm{v}}} \text { and }(\mathrm{w}, \mathrm{y}) \in \phi(\mathrm{G}, \mathrm{H})\right\}
\end{aligned}
$$

\} while $\left(\mathrm{G}_{\mathrm{u}} \neq \varnothing\right.$ and $\left._{\mathrm{v}} \neq \varnothing\right)$

In terms of algorithmic complexity, observe that during the depth-first construction of the matching chains, each vertex in $\mathrm{G}$ or $\mathrm{H}$ will be matched atmost once in the forward procedure. Once a vertex is mapped, it will never participate in another mapping again. The total time complexity of constructing the matching chains is therefore bounded by $\mathrm{O}\left(\mathrm{n}^{2} \sqrt{\mathrm{nlog} \log \mathrm{n}}\right)$, for $\mathrm{n}=\max (\mathrm{n} 1, \mathrm{n} 2)$. Moreover, the construction of the $\chi(v)$ vectors will take $\mathrm{O}(\mathrm{n} \sqrt{\mathrm{nL}})$ time, implying that the overall complexity of the algorithm is $\max \left(O\left(n^{2} \sqrt{n \log \log n}\right), O\left(n^{2} \sqrt{n L}\right)\right)$.

\subsection{Example}

To illustrate the matching algorithm, we consider the two shock trees shown in Fig 3.2 (top), each of which describes a different view of a brush. The underlying shocks, along with the final computed correspondences between nodes, are depicted in Fig 3.2 (bottom). The sequence of steps in finding this best correspondence (minimum-weight maximum cardinality matching) between the two shock trees is shown in Fig 3.3.Briefly each step in the sequence are described.

Steps 1-4:

The algorithm finds the minimum weight matching between the two shock trees, seeking to find the two subtrees which are maximally similar in terms of their topological structure and the geometry of their root nodes (shocks). In this example, the two subtrees rooted at 1-007 and 1-005 (denoted by bold circles in Fig 3.3) are selected as most similar. In step 2, this pair is added to the set of final correspondences (denoted by short-dashed circles) and the algorithm is recursively applied to the subtrees of 1-007 and 1-005. In this manner, the correspondences (3-001,3-002) and (1-003,1-001) are added to the set of final correspondences.

Steps 5-6:

After descending to the bottom of the subtrees rooted at (1-007,1-005), control is returned to (1-007,1-005) and these two subtrees are removed from the original shock graphs. From the resulting shock subtrees, we repeat the process of finding the best corresponding subtrees. In step 5, the subtree pair (1-006,1-004) is selected and added to the final correspondences in step 6.

Steps 7-12:

After removing the subgraphs originating at (1-006,1-004), a new pair (3-002,3-001) is selected in step 7 and added to the final correspondences in step 8. After removing this newpair, the process is applied to the remaining shock forests in step 9, resulting in the selection of the pair (1-004,1-002). This pair is added to the final correspondences in step 10. In step 11, the pair (1-005,1-003) is selected and added to the final 
correspondences in step 12. The algorithm terminates by leaving the nodes 1-001 and 1-002 as unmatched vertices (denoted by long-dashed circles) in the shock tree corresponding to leftmost object in Fig 3.2 (bottom).

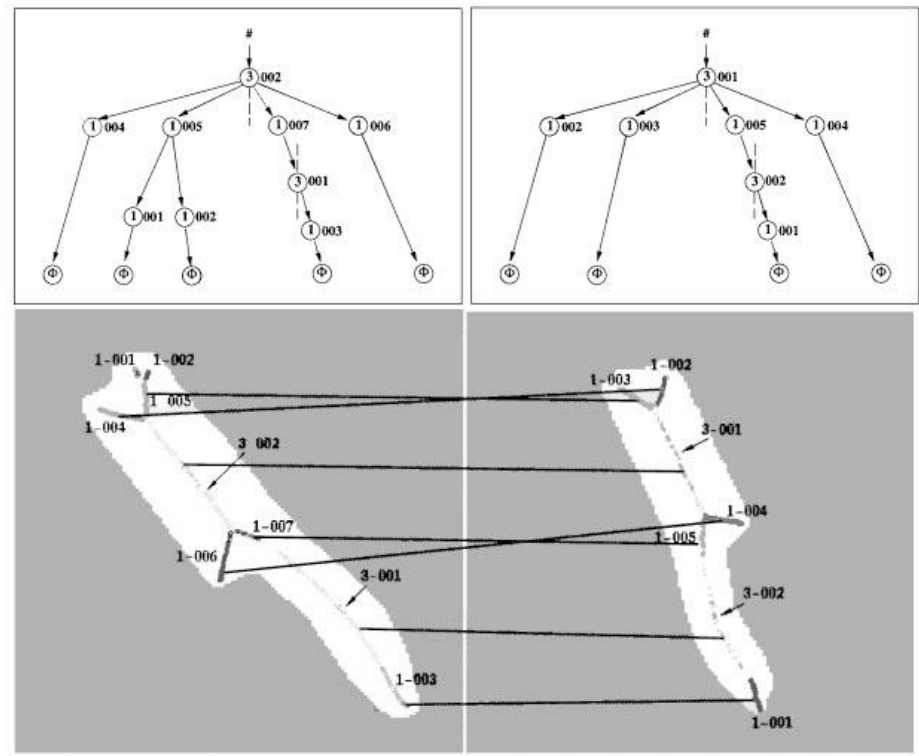

Fig 3.2

Top: The shock trees derived for two different views of a brush.

Bottom: The correspondences between nodes in the shocktrees computed by the matching algorithm.
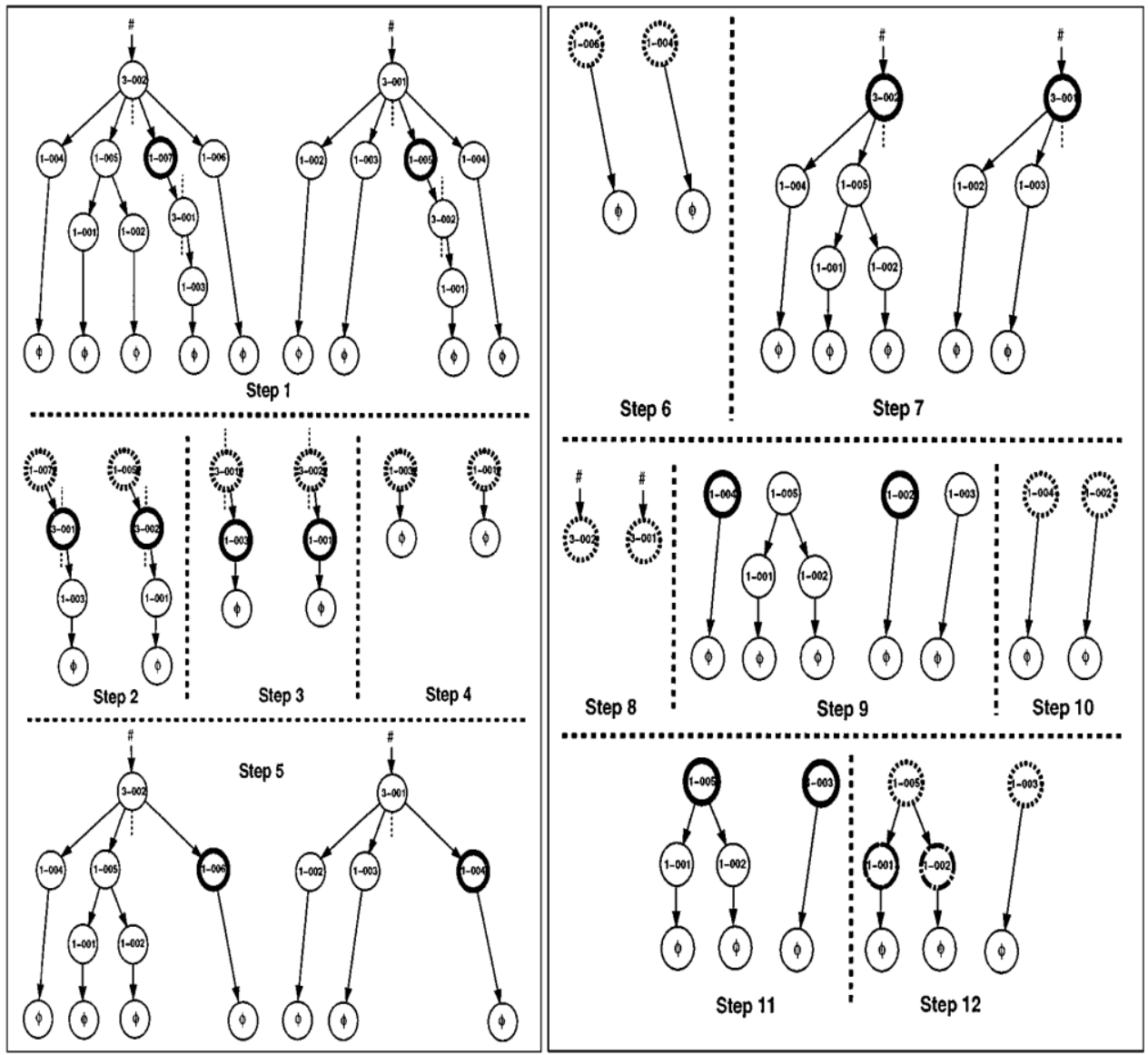

Fig 3.3

Step-by-step execution of the matching algorithm applied to the shock trees in Fig. 3.3. The roots of subtrees selected as most similarare denoted by bold circles. These are subsequently added to the set of final correspondences (short-dashed circles). Unmatched nodes aredenoted by long-dashed circles. 


\section{Conclusion}

This paper deals with a generic description of object shape, based on the singularities of a curve evolution equation derived from the object's boundary, can be organized into a graph representation and how this graph can be matched into an object database. The rules of the shock graph grammar, conversion of shock graph to unique rooted shock tree and algorithm for finding best set of corresponding nodes between two shock trees in polynomial time have been discussed.

\section{References}

[1]. Alizadeh, F. 1995. Interior point methods in semidefinite programming with applications to combinatorial optimization. SIAM J.Optim., 5(1):13-51.

[2]. Alvarez, L., Lions, P.L., and Morel, J.M. 1992. Image selective smoothing and edge detection by nonlinear diffusion.SIAM J. Numer. Anal., 29:845-866.

[3]. Arnold, V. 1991. The Theory of Singularities and Its Applications.LezioniFermiane, Piza, Italy.

[4]. Basri, R., Costa, L., Geiger, D., and Jacobs, D. 1995. Determining the similarity of deformable shapes.In Proc. ICCV Workshop on Physics-Based Modeling in Computer Vision, pp. 135-143.

[5]. Basri, R. and Ullman, S. 1988. The alignment of objects with smooth surfaces.In Second International Conference on Computer Vision (Tampa, FL, December 5-8, 1988), Computer Society Press: Washington, DC, pp. 482-488.

[6]. Blum, H. 1973. Biological shape and visual science.J. Theor. Biol., 38:205-287.

[7]. Bulthoff, H.H. and Edelman, S. 1992. Psychophysical support for a two-dimensional view interpolation theory of object recognition.Proc. of the National Academy of Sciences, 89:60-64.

[8]. Burbeck, C.A. and Pizer, S.M. 1995. Object representation by cores: Identifying and representing primitive spatial regions. Vision Research, 35:1917-1930.

[9]. Burns, J. and Kitchen, L. 1987. Recognition in 2D images of 3D objects from large model bases using prediction hierarchies. In Proc. International Joint Conference on Artificial Intelligence, Milan, Italy, pp. 763-766.

[10]. Cootes, T.F., Hill, A., Taylor, C.J., and Haslam, J. 1996. The use of active shape models for locating structures in medical images. In Fourth European Conference on Computer Vision, Cambridge, UK.

[11]. Overton, M.L. and Womersley, R.S. 1993. Optimality conditions and duality theory for minimizing sums of the largest eigen values of symmetric matrices. Math. Programming, 62(2):321-357.

[12]. Pauwels, E., Moons, T., Gool, L.J.V., Kempenaers, P., and Oosterlinck, A. 1995. Recognition of planar shapes under affine distortion.International Journal of Computer Vision, 14(1):49-65.

[13]. Pelillo, M., Siddiqi, K., and Zucker, S.W. 1998. Matching hierarchical structures using association graphs.In Fifth European Conference on Computer Vision, vol. 2, Freiburg, Germany, pp. 3-16.

[14]. Pizer, S.M., Fritsch, D.S., YushKevich, P.A., Johnson, V.E., and Chaney, E.L. 1999. Segmentation, registration and measurement of shape variation via image object shape. IEEE Transactions on Medical Imaging, Special Issue on Model-Based Analysis of Medical Images. (to appear)

[15]. Pizer, S.M., Oliver, W.R., and Bloomberg, S.H. 1987. Hierarchical shape description via the multiresolution symmetric axis transform. IEEE Transactions on Pattern Analysis and Machine Intelligence, 9(4):505-511. 